\title{
New Seismic Attenuation System (SAS) for the Advanced LIGO Configurations (LIGO2)
}

\author{
Alessandro Bertolini ${ }^{1}$, Giancarlo Cella ${ }^{1}$, Erika D'Ambrosio ${ }^{2}$, Riccardo \\ DeSalvo $^{2}$, Virginio Sannibale ${ }^{2}$, Akiteru Takamori ${ }^{3}$, Hiroaki Yamamoto ${ }^{2}$ \\ I. Universita' di Pisa, \\ II. California Institute of Technology, \\ piazza Torricelli 2, $\quad 56100-$ Pisa, Italy \\ III. Tokyo University, \\ 1200 E. California Bl. Pasadena, CA 91125, USA \\ 7-3-1 Hongo Bunkyo-ku Tokyo 113-0033, Japan
}

\begin{abstract}
A new passive seismic attenuation system is being developed to replace the current passive attenuation stacks in LIGO 2, it is expected to drive the seismic contribution to the interferometer noise below any other noise source. The SAS will be effective completely starting at about $5 \mathrm{~Hz}$, well inside the (uncompensated) gravity gradient noise wall.
\end{abstract}

\section{INTRODUCTION}

The SAS is a passive chain of low frequency attenuation filters preceded by an Ultra Low Frequency (ULF), passive and active pre-filtering stage and followed by a multiple pendulum mirror suspension system. The multiple pendulum controls are performed, like in $\mathrm{Virgo}^{1}$ and $\mathrm{GEO}^{2}$ from recoiling masses. These masses are suspended, together with the mirror and its upper bobs, from the SAS last filter. The two top multiple pendulum masses are expected to have a GEO-like configuration and would be acted on, like in the Virgo "marionetta", from a rigid structure mounted on the last SAS filter that supports suitable electromagnetic control actuators. The multiple pendulum configuration, on top of allowing better control of suspension thermal noise, is expected to allow better control authority distribution to lighten, or perhaps virtually eliminating, the control load of the mirror actuators. To this end it is also necessary to deliver a very low level of residual r.m.s. motion which is obtained by means of the ULF pre-filtering stage and its active inertial damping of resonances ${ }^{3,4,5}$.

The ULF stage, tuned well below $100 \mathrm{mHz}$, has the triple function to provide:

- Passive attenuation in frequency regions of the micro-seismic peak, where the passive filter chain has no attenuation capabilities, and of the passive filters internal resonances.

- DC and ULF positioning and control.

- A soft support for the active inertial damping of the SAS passive filters resonances and residual r.m.s. motion reduction.

CP523, Gravitational Waves: Third Edoardo Amaldi Conference, edited by S. Meshkov (C) 2000 American Institute of Physics 1-56396-944-0/00/\$17.00 

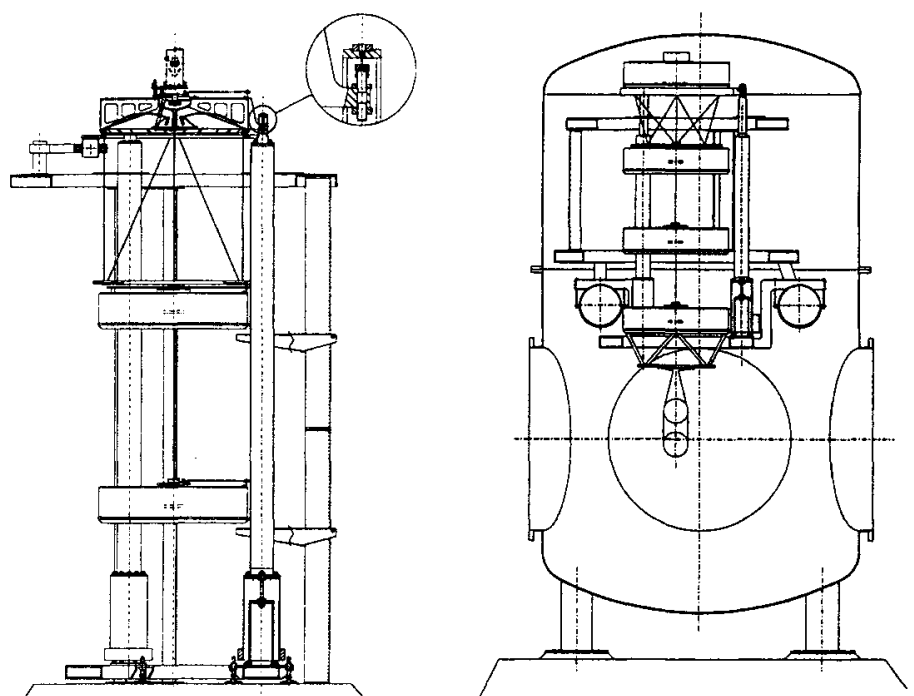

FIGURE 1. Present prototype of SAS (left) and tentative design to fit within the LIGO BSC chambers (right). The Filter Zero (at the top of the figure) is supported by three IP legs bolted on a rigid platform fixed on ground. The soft hinges (insert) allow full $x, y$ and $\phi$ movement of the IP. The IP is controlled by actuators and position sensors (one shown on the left top corner) mounted on a rigid safety/reference structure. It will also carry horizontal and vertical accelerometers for inertial active damping of resonances. The passive chain suspension wire hangs down from the center of F0. F0 also carries a disk, suspended by means of an hexa-wire, loaded with magnets which, generating eddy current on the first passive filter surface, provide effective velocity damping of all resonances. Two or three passive filters (see figure 2) carry the payload. The triple pendulum, visible in the BSC assembly on the right, is suspended and acted on from the bottom filter body.

\section{GEOMETRICAL ANTI SPRING FILTERS (GASF)}

The core of SAS is the Geometric Anti Spring Filter (GASF) ${ }^{6}$. In a GASF low frequency vertical oscillations are obtained by directing part of the cantilever suspension springs return forces in an antagonist way that reduces the effective vertical spring constant without significantly reducing the necessary suspension force. The effective spring constant, and hence the vertical resonant frequency can be tuned at will, conceptually down to zero, by adjusting the level of spring antagonism. As a result a low and stable vertical resonant frequency can be factory tuned. The chosen frequency varies little with temperature and vertical working point choice; as a result an extremely large linearity is obtained with negligible up-conversions even with large oscillation excitations. Also the GASF require no precise tuning of their vertical working point.

Passive attenuation in the other five degrees of freedom is obtained by standard low frequency pendulum action? 


\section{Creep and Creak Immunity}

Great care is taken in the GASF design to eliminate from the stress path any joint subject to shear to eliminate creak noise ${ }^{8}$.

In all the three joints, between the suspension wires and the filter bodies, and at the two ends of the cantilever suspension blades, the stress is perpendicular (or quasi perpendicular) to the contact surfaces and a low melting alloy coating will braze these joints together during the filter's bake-out. To avoid internal noise production we also eliminated all helicoidal springs that, on top of their low frequency resonances, present problems of stress concentration or stick and slip at their extremities.

Frozen dislocation materials (precipitation hardened alloys like Maraging Steel ${ }^{9}$ ) used at carefully controlled stress levels are used to eliminate the creep noise from highly stresses components (cantilever blade springs and suspension wires) while bonded steel alloys are used in all low stress parts.

Additionally a bake-out at full load is foreseen to burn out all residual dislocation and potential creep noise before operations. This same bake-out procedure will performed the brazing of all the joints under stress and clean-out the filters for Ultra High Vacuum (UHV) operation. The simplicity of the filter's structure and it's all metal nature makes it easy to clean them to UHV requirements.

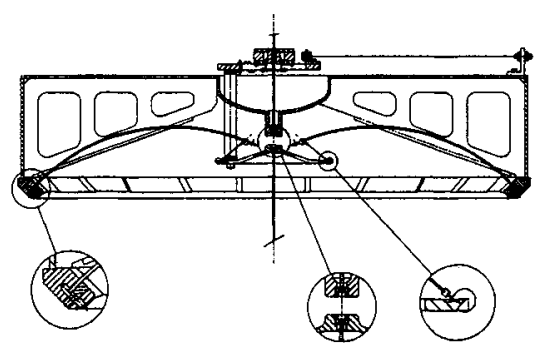

FIGURE 2. Standard Filter design. The filter body is suspended from a suspension wire, it carries an even number of prestressed cantilever spring blades (the ached components) which work in partially antagonist way. This configuration allows an easy tuning of the vertical resonant frequency. All contacts between the filter components (see inserts) are made with zero or negligible efforts in the contact planes to avoid creak noise.

\section{Some SAS Technical Advantages}

SAS, being a passive system is stable even in case of power failure. The great softness and extremely large geometrical dynamic range of its ULF stage ${ }^{10}$ allows essentially unlimited operation using very low in vacuum power levels to compensate for tidal and other daily movements of the interferometer baseline. Additionally these control forces are applied very far from the payload, thus minimizing possible thermal perturbations to the mirror suspensions.

SAS is designed as a self supporting, transportable unit that can rapidly installed in the existing LIGO vacuum vessels ${ }^{11,12}$. Additionally SAS has large payload 
capabilities (more than 0.5 tons without modifications) and two independent SAS chains can be mounted inside the existing LIGO BSC inner diameter.

\section{SAS ADVANCEMENT STATUS}

Individual GASF and the behavior of complete chains have been simulated and complete engineering design have been produced.

UHV grade GASF and Inverted Pendulum prototypes have already been built.

The attenuation performances of both isolated GASFs and doublets have been partially tested. The measured behavior perfectly matched the earlier simulated one thus validating the simulations of longer chains. These simulations show that SAS can generate seismic attenuation crossing the mirror thermal noise floor below $6 \mathrm{~Hz}$, exceeding the $10 \mathrm{~Hz}$ LIGO 2 specifications by such a margin to give good confidence that the seismic noise will not be a limiting factor.

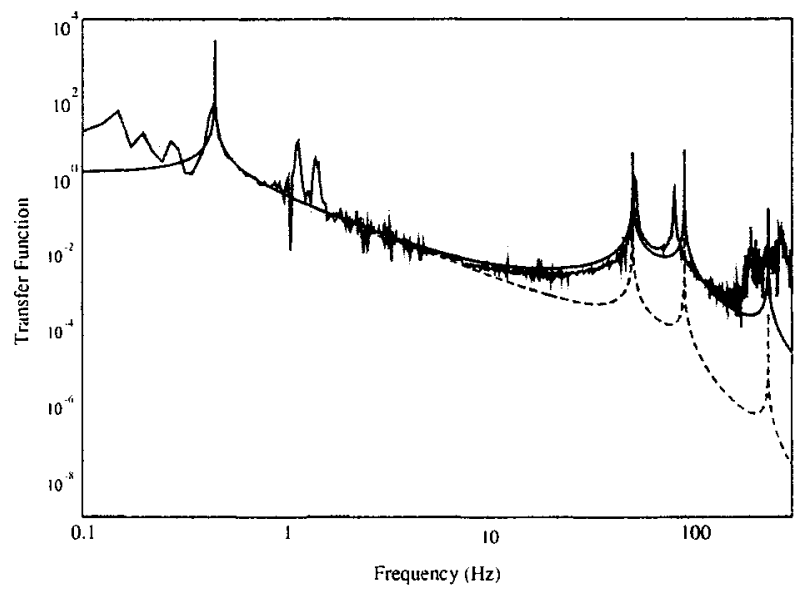

FIGURE 3. Attenuation performance of a single standard filter. Please note the agreement between simulation (red) and later measured data (black). The two peaks above $1 \mathrm{~Hz}$ are payload tilt resonances. In this measurement the mode dampers designed to eliminate the peaks at 55 and $80 \mathrm{~Hz}$ have not been implemented yet. The measurement is instrument noise dominated above $150 \mathrm{~Hz}$.

The fact that SAS is effective at such low frequencies that gravity gradient noise aiready dominates may seem a waste; however this frequency domain overkill comes for free and shields the test mass from possible up-conversions and nonlinear effects from the control system and transient perturbations. Additionally it may come useful if gravity gradient noise cancellation techniques were to prove viable.

The best performance of SAS are obtained with tall towers, still fitting below the LIGO hall ceiling. The vacuum vessel for these towers would be obtained introducing a barrel extension between the BSC body and its hat. Even a scaled down SAS version, fitting inside the present short vacuum envelopes, largely outperforms any other proposed seismic attenuation scheme and, thanks to the SAS modularity, can later on be extended to the high performance tall tower configuration with simple addition of units and replacement of elastic joints and Inverted Pendulum legs. 
High sensitivity accelerometers are being developed for the SAS inertial damping ${ }^{13}$.
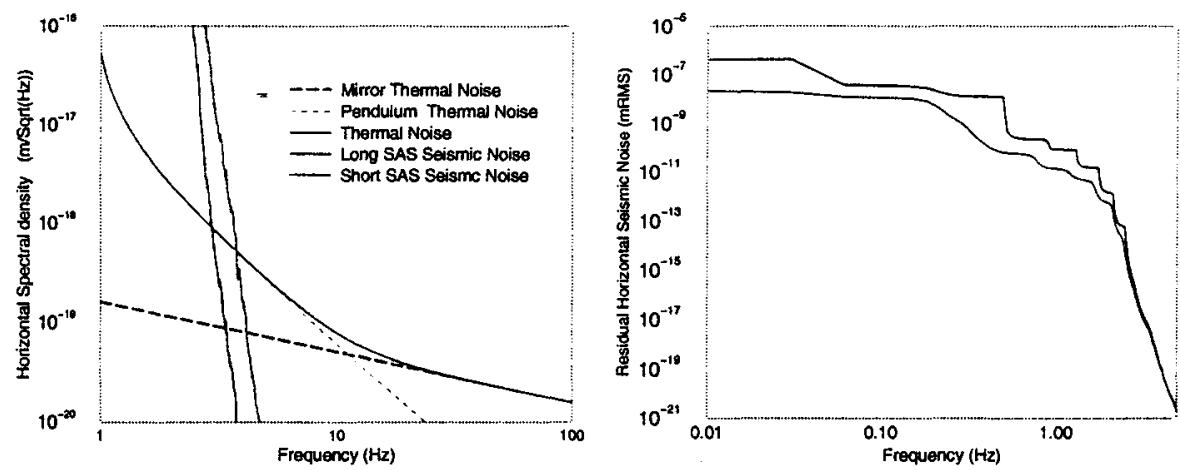

FIGURE 4. Simulated performance of the SAS chains: residual mirror seismic noise on the left and integrated residual r.m.s. motion on the right. In both cases the ground seismic activity of the Livingstone site have been used as an input to the simulation. The short SAS simulation corresponds to the system of figure 2. The long SAS simulation is for a longer chain mounted in a BSC extension barrel designed for rapid installation procedures. The integrated r.m.s. motion on the right is calculated for the short chain.

\section{ACKNOWLEDGMENTS}

We like to acknowledge the enthusiastic contribution of Henry Lubatti, David Ahkavan and Nicolas Viboud, students that recently joined the SAS group. We also acknowledge the professionality of Ricardo Paniagua and his crew of the Caltech machine shop, Gianni Gennaro, master designer and Carlo Galli, passionate builder of the SAS system.

\section{REFERENCES}

1. Virgo Collaboration VIRGO final design report. VIR-TRE-1000-13 (May 1997)

2. M.V. Plissi, et al., Rev. Sci. Instrum., 69, 3055-3061, 1998

3. G. Losurdo: Thesis: "Ultra-low Frequency Inverted Pendulum for the Virgo Test Mass Suspension", Scuola Normale Superiore di Pisa, Classe di Scienze, 1998.

4. G Losurdo, et al. "Inertial control of the VIRGO Superattenuator", this conference

5. G. Losurdo, et al: Review of Scientific Instruments, 70, 2507, 1999.

6. A. Bertolini, at al., Nucl. Instr. and Meth. in Phys. Res. A 4353 (1999) pp. 475-483.

7. M. Beccaria, et al: Nucl. Instr. and Meth. in Phys. Res. A, 397, 1999.

8. R DeSalvo, et al., conference proceedings, Moriond rencontres ... January 1999.

9. S. Braccini, et al., Nucl. Instr. and Meth. in Phys. Res., A : 404, 455-479 - (1998).

10. R. DeSalvo, et al: Nucl. Instr. and Meth. in Phys. Res. A 420, 316, 1999.

11. M. Barton, et al.: "Proposal of a Seismic Attenuation System for ...", Note, LIGO-T990075-00-D

12. M. Barton, et al.: "Answers to 'Isolation criteria for the LIGO II...' ", Note, LIGO-T990076-00-D

13. A.Bertolini, et al., "High sensitivity accelerometers for high performant seismic attenuators", this conference. 